\title{
Relaxation de contraintes et fissurations induites par une redistribution d'eau en milieu biphasique élastique
}

\author{
IBRAHIM Mrani ${ }^{1}$ ET JEAN-ClaUde BÉNET ${ }^{2, a}$ \\ 1 Faculté des sciences, département de physique, El Jadida, Maroc \\ 2 Laboratoire de Mécanique et Génie Civil, Université de Montpellier II, Cc 48, 34095 Montpellier Cedex 5, France
}

Reçu le 5 décembre 2003, accepté le 5 décembre 2004

\begin{abstract}
Résumé - À partir d'un modèle de transport d'eau en milieu biphasique et élastique [I. Mrani, J.-C. Bénet, G. Fras, Transport of water in a biconstituant elastic medium, Appl. Mech. Rev., special issue on Mechanics of swelling 48 (1995) 717-721], on examine dans cette étude les risques de fissurations induites par la redistribution d'eau dans un échantillon de forme cylindrique après séchage. La résolution numérique de l'équation de transport de l'eau et de l'équation d'équilibre mécanique donnent accès à l'évolution de la teneur en eau, de la déformation et des contraintes dans le milieu. On s'intéressera à l'état du matériau pendant les phases de séchage, de refroidissement et de stockage. À l'issue de cette étude, les risques de fissuration sont analysés pour différents scénarios de séchage, qui déterminent l'évolution de l'état hydrique pendant le stockage.
\end{abstract}

Mots clés : Milieu déformable / transport d'eau / déformations / contraintes / fissurations / simulation numérique

\begin{abstract}
Stress relaxation and cracking induced by a water redistribution in elastic biphasic medium. From a model of transport of water in elastic medium [I. Mrani, J.-C. Bénet, G. Fras, Transport of water in a biconstituant elastic medium, Appl. Mech. Rev., special issue on Mechanics of swelling 48 (1995) 717-721] the risk of craking induced by the water redistribution in a cylinder sample after drying is analysed. The numerical resolution of the transport equation of water and the mechanical equilibrium equation give access to the evolution of the water content, the deformation and the stresses in the medium. One will take interest in the state of material during drying, cooling and storage phases. At the end of this study, the risks of cracking are analyzed for various configurations of drying wich determine the hydrous state evolution during storage.
\end{abstract}

Key words: Deformable medium / water transport / deformations / stresses / cracking / numerical simulation

\section{Introduction}

La phase de séchage d'un matériau est accompagnée le plus souvent par une phase de retour de la température ambiante et une phase de stockage au cours de laquelle le matériau est conditionné de façon à ce qu'aucun échange hydrique ou thermique avec l'extérieur ne se produise. Pendant la phase de séchage, le départ d'eau est accompagné de contraintes, qui dans le cas où elles dépassent les contraintes de rupture du matériau provoquent sa fissuration. Pendant les phases de refroidissement et de stockage, il peut y avoir modification des contraintes sous l'action de la redistribution de la teneur en eau ce qui crée des zones de tensions dans le milieu pouvant à leurs tours être à l'origine de fissurations.

\footnotetext{
a Auteur correspondant : benet@lmgc.univ-montp2.fr
}

À partir d'un modèle existant [1,2], on se propose de décrire et prévoir le phénomène de la fissuration du milieu au cours du séchage et de la phase de stockage. L'analyse de la dégradation du matériau est basée sur l'étude de la redistribution des profils de teneur en eau en fin de séchage et la relaxation des contraintes qui en découle.

L'étude est divisée en deux parties :

- Dans une première partie, on présente l'étude expérimentale des propriétés hygroscopiques, des propriétés de transport d'eau et des propriétés mécaniques du matériau utilisé et ce en fonction de l'évolution de la température et de la teneur en eau. Le matériau utilisé est une pâte alimentaire diphasique.

- Dans la deuxième partie, on aborde la simulation numérique du séchage d'un cylindre en utilisant différents diagrammes de séchage. L'analyse de ces 


\section{Nomenclature}

\begin{tabular}{|c|c|}
\hline$\beta$ & Coefficients d'expansion hydrique \\
\hline$\beta_{n}$ & nième caractéristique des fonctions de Bessel de première espèce et d'ordre 0 \\
\hline$\delta_{i j}$ & Tenseur unité \\
\hline$\epsilon_{i j}$ & Tenseur des déformations \\
\hline$\lambda$ et $\mu$ & Coefficients de Lamé Pa \\
\hline$\rho_{\mathrm{e}}^{*}$ & Masse volumique de l'eau $=10^{3} \mathrm{~kg} \cdot \mathrm{m}^{-3}$ \\
\hline$\rho_{\mathrm{s}}$ & Masse volumique apparente du solide $\mathrm{kg} \cdot \mathrm{m}^{-3}$ \\
\hline$\sigma_{\theta \theta}$ & Contrainte circonférentielle $\mathrm{Pa}$ \\
\hline$\sigma_{i j}$ & Tenseur des contraintes $\mathrm{Pa}$ \\
\hline$\sigma_{r r}$ & Contrainte radiale $\mathrm{Pa}$ \\
\hline$\sigma_{r z}$ & Contrainte de cisaillement $\mathrm{Pa}$ \\
\hline$\sigma_{r}$ & Contrainte à la rupture $\mathrm{Pa}$ \\
\hline$\sigma_{z z}$ & Contrainte axiale $\mathrm{Pa}$ \\
\hline $\overrightarrow{\mathbf{n}}$ & Vecteur normal aux frontières externes du milieu \\
\hline$a_{\mathrm{w}}$ & Activité de l'eau $a_{\mathrm{w}}=H R / 100$ \\
\hline$D$ & Coefficient de diffusion de l'eau $\mathrm{m}^{2} \cdot \mathrm{s}^{-1}$ \\
\hline$E$ & Module de Young Pa \\
\hline$h(t)$ & Hauteur de l'élément cylindrique à l'instant $t \mathrm{~m}$ \\
\hline$H R$ & Humidité relative de l'air \% \\
\hline$J$ & Flux radial de transport de l'eau kg.m ${ }^{2} . \mathrm{s}^{-1}$ \\
\hline$K$ & Coefficient de compressibilité $\mathrm{Pa}$ \\
\hline$R(t)$ & Rayon du cylindre à l'instant $t \mathrm{~m}$ \\
\hline$R_{\mathrm{c}}$ & Rayon du cylindre de matériau m \\
\hline$u_{r}$ et $u_{z}$ & Déplacements radial et axial $\mathrm{m}$ \\
\hline$w$ & Teneur en eau \\
\hline$w^{0}$ & Teneur en eau initiale \\
\hline$w_{\mathrm{e}}$ & Teneur en eau à l'équilibre \\
\hline$w_{\text {surf }}$ & Teneur en eau à la surface du milieu \\
\hline
\end{tabular}

résultats permettra de conclure sur les risques de fissuration pendant le séchage et lors du stockage.

\section{2 Équations du modèle}

On adopte une description macroscopique où le milieu est considéré comme la superposition de deux milieux continus interagissant entre eux : le squelette solide et le liquide qui « diffuse » à l'intérieur du solide. Le modèle est développé dans le cadre des hypothèses suivantes :

- le milieu est biphasique sans apparition de phase gazeuse ;

- le squelette solide est isotrope, élastique dans le cadre de déformations infinitésimales ;

- la température est uniforme et constante;

- le matériau est inerte et ne donne lieu à aucune réaction chimique.
Les variables d'état utilisées par la suite sont la teneur en eau $w$ et le tenseur des déformations $\epsilon_{i j}$.

\section{1 Équation de transport de l'eau}

Le modèle est limité ici au cas d'une géométrie cylindrique de longueur infinie par rapport à son rayon $r$. le transport d'eau s'effectue essentiellement dans le sens radial du milieu. Dans ces conditions, l'équation de transport d'eau s'écrit [3] :

$$
\frac{\partial w}{\partial t}=\frac{1}{r} \frac{\partial}{\partial r}\left(r D \frac{\partial w}{\partial r}\right)
$$

\section{2 Équations d'état et d'équilibre}

Les équations d'état définissent le comportement mécanique du matériau au cours d'une transformation 
quelconque. Autour d'un état de référence, l'évolution incrémentale du tenseur de contrainte $\sigma_{i j}$ est définie en fonction des variations incrémentales des variables d'état, la teneur en eau $w$ et le tenseur de déformations $\epsilon_{i j}[3]$ :

$$
\delta \sigma_{i j}=\lambda \delta(\operatorname{tr} \epsilon) \delta_{i j}+2 \mu \delta \epsilon_{i j}-3 K \beta \delta w \delta_{i j}
$$

où $\delta \Psi$ désigne une variation incrémentale de la grandeur $\Psi$.

La conservation de la quantité de mouvement totale du milieu durant des transformations quasi-statiques, dans lesquelles la gravité et les accélérations du liquide et du solide sont négligées, se traduit par l'équation d'équilibre :

$$
\operatorname{div} \sigma_{i j}=0
$$

\section{3 Étude expérimentale des propriétés physiques et mécaniques du matériau}

\subsection{Isotherme de désorption}

Les expériences consistent à réaliser des équilibres thermodynamiques dans une enceinte régulée en température et en humidité. Les équilibres couvrent une plage de température allant de $30{ }^{\circ} \mathrm{C}$ à $90{ }^{\circ} \mathrm{C}$ et une plage d'humidité relative allant de $40 \%$ à $90 \%$. L'exploitation des différents résultats obtenus a permis d'obtenir les isothermes de désorption de la figure 1. Une modélisation de ces courbes est donnée par l'équation suivante :

$$
w=\left(p_{1} T+p_{2}\right)\left[\frac{a_{\mathrm{w}}}{1-a_{\mathrm{w}}}\right]^{\left(p_{3} T+p_{4}\right)}
$$

$p_{1}=-0,134 p_{2}=15,5 p_{3}=9,55 \times 10^{-3} p_{4}=-8,3 \times 10^{-2}$ où $w$ est la teneur en eau d'équilibre en $\%$.

On notera une grande cohérence des isothermes; ce qui tend à montrer l'homogénéité des échantillons et la fiabilité des techniques expérimentales. Ces isothermes sont conformes aux résultats de la littérature [4].

\subsection{Propriétés de retrait}

Pour un matériau de structure diphasique (eau + matière solide), l'additivité des volumes conduit à l'expression du coefficient d'expansion hydrique $\beta$ [5] par :

$$
\beta=\frac{1}{3(\alpha+w)}
$$

où $\alpha$ est le rapport entre la masse volumique de l'eau et celle du constituant de la phase solide. Dans le cas du matériau utilisé $\alpha=0,6923$.

La masse volumique apparente de la phase solide s'exprime par :

$$
\rho_{\mathrm{s}}=\frac{\rho_{\mathrm{e}}^{*}}{\alpha+w}
$$

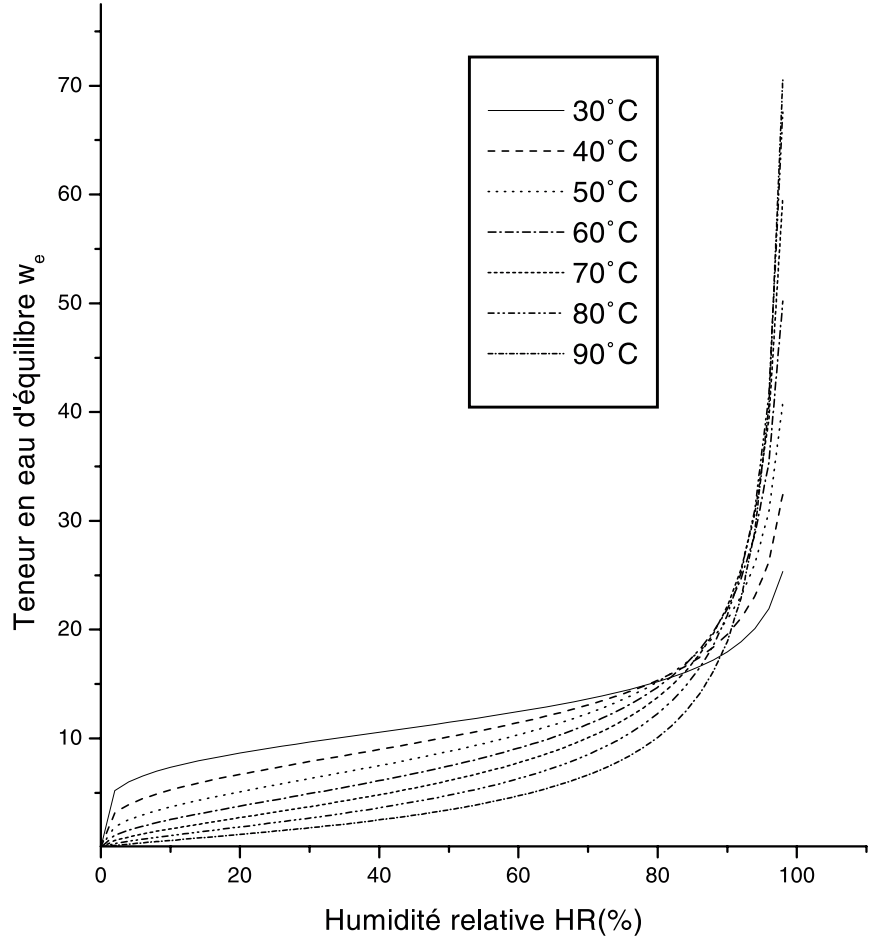

Fig. 1. Isothermes de désorption.

\subsection{Coefficient de transport de l'eau}

Dans le cas de l'hypothèse de faible gradient de $w$, la solution de l'équation (1) est de la forme [6] :

$$
\frac{w-w_{\mathrm{e}}}{w^{0}-w_{\mathrm{e}}}=\sum_{n=1}^{+\infty}\left[\frac{4}{\beta_{n}^{2}} \exp \left(-\beta_{n}^{2} \frac{D t}{R_{\mathrm{c}}^{2}}\right)\right]
$$

où $w$ est la teneur en eau moyenne à l'instant $t$.

Afin de respecter les conditions de validité de (5), à partir d'un état d'équilibre caractérisé par un gradient de $w, \rho_{\mathrm{s}}$ et $D$ très faible, on provoque, par l'intermédiaire d'une atmosphère régulée un échelon de teneur en eau $\left(w_{0}-w_{\mathrm{e}}\right)$ avec $\left|w_{0}-w_{\mathrm{e}}\right| \ll w_{0}$. La pesée en continu d'un échantillon de matériau entre deux états d'équilibre $w_{0}$ et $w_{\mathrm{e}}$ fournit le coefficient $D$ par l'intermédiaire de (5) limitée au premier ordre. Le coefficient calculé est affecté à la teneur en eau $w_{0}$. La répétition de l'expérience pour des températures comprises entre $30{ }^{\circ} \mathrm{C}$ et $90{ }^{\circ} \mathrm{C}$ et des teneurs en eau comprises entre $10 \%$ et $40 \%$ conduit à la figure 2, qui montre l'évolution de $D$ en fonction de $w$.

L'ensemble des expériences réalisées a montré que la diffusivité $D$ varie très peu en fonction de la température. Il a été opté pour un lissage de l'évolution du coefficient $D$ en fonction de la teneur en eau uniquement :

$$
D=\frac{p_{1}-p_{2}}{1+\exp \left(\frac{w-w_{0}}{\mathrm{~d} w}\right)}+p_{2} \quad\left(\times 10^{-11} \mathrm{~m}^{2} \cdot \mathrm{s}^{-1}\right)
$$

avec $: p_{1}=0,70805, p_{2}=23,007, w_{0}=33,529, \mathrm{~d} w=$ 5,9998 . 


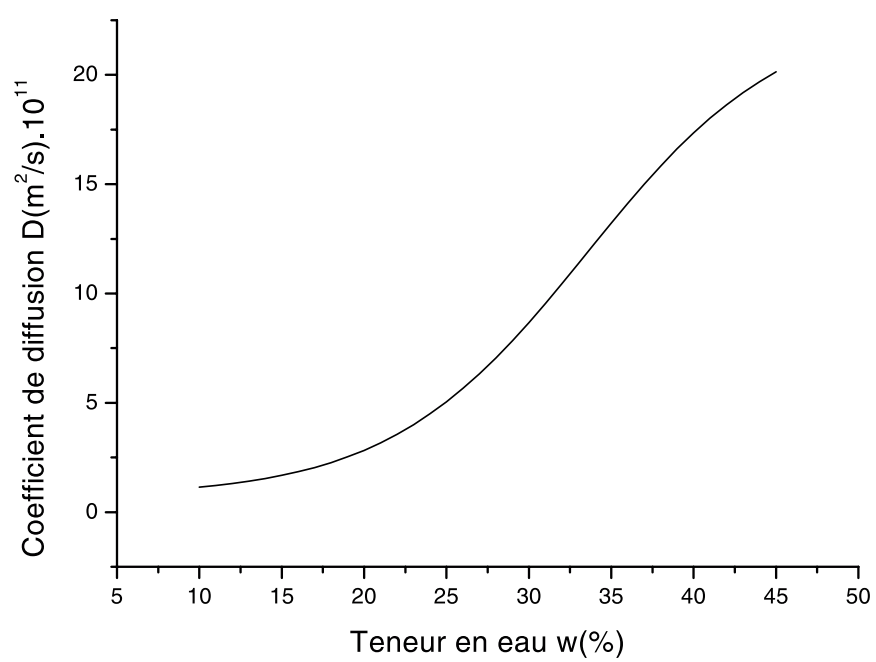

Fig. 2. Évolution du coefficient de diffusion en fonction de la teneur en eau.

\subsection{Propriétés mécaniques}

\subsubsection{Dispositif expérimental}

Les essais mécaniques ont été réalisés sur un rhéomètre RHEO TAXT 2/5 de capacité 5 daN (Fig. 3). À l'état initial, les échantillons de section cylindrique sont placés dans l'enceinte A conditionnée en temperature $T$ et humidité $H R$. La valeur de $H R$ est choisie pour amener le produit à la teneur en eau souhaitée conformément à l'isotherme de désorption (Fig. 1). Cette phase dure environ 24 heures. À l'équilibre 10 échantillons sont prélevés, coupés et munis de deux embouts métalliques collés et replacés ensuite dans l'enceinte A. Cette opération dure environ $15 \mathrm{~s}$ pour chaque éprouvette réalisée. Les éprouvettes sont maintenues dans l'enceinte A pendant $30 \mathrm{~min}$ de façon à ce qu'elles reviennent à l'équilibre. On effectue ensuite des essais mécaniques sur les 10 éprouvettes afin de s'assurer de la répétabilité de l'essai. L'essai mécanique dure de 10 à 15 s et on peut supposer que les caractéristiques hydriques et la température ne varient pas au cours de cet essai. Les courbes de traction permettent de déterminer le module d'élasticité et la contrainte de rupture.

\subsubsection{Module d'élasticité}

Les expériences de chargement mécanique ont été réalisées pour des températures allant de $50{ }^{\circ} \mathrm{C}$ à $90{ }^{\circ} \mathrm{C}$ et pour des humidités relatives allant de $40 \%$ à $90 \%$, de façon à couvrir une large gamme de teneur en eau. L'exploitation de l'ensemble de ces essais a montré que le module de Young du matériau varie très peu en fonction de la température conformément à la littérature [4]. Une courbe moyenne de l'évolution de ce coefficient en fonction de la teneur en eau est présentée sur la figure 4. La corrélation mathématique de l'évolution du module de
Young en fonction de la teneur en eau est donnée par la sigmoïde suivante :

$$
E=\frac{p_{1}-p_{2}}{1+\exp \left(\frac{w-w_{0}}{\mathrm{~d} w}\right)}+p_{2}
$$

avec : $p_{1}=7,1557, p_{2}=0,08067, w_{0}=6,0205, \mathrm{~d} w=$ 4,9237 .

\subsubsection{Détermination de la contrainte à la rupture}

Les essais mécaniques de traction permettent également de déterminer la contrainte à la rupture de l'échantillon. Cette contrainte est déduite des courbes de chargement qui définissent l'évolution de la contrainte en fonction de la déformation de l'échantillon. L'ensemble des valeurs montre que celle-ci varie peu en fonction de la température. Nous avons présenté son évolution en fonction de la teneur en eau sur la figure 5.

La corrélation mathématique de l'évolution de la contrainte à la rupture en fonction de la teneur en eau est donnée par la sigmoïde suivante :

$$
\sigma_{r}=\frac{p_{1}-p_{2}}{1+\exp \left(\frac{w-w_{0}}{\mathrm{~d} w}\right)}+p_{2}
$$

avec : $p_{1}=15,159, p_{2}=0,3768, w_{0}=10,187, \mathrm{~d} w=$ 4,2057 .

\section{Simulation numérique du séchage du matériau}

\subsection{Définition du domaine d'étude}

Le matériau de géométrie cylindrique est placé dans une atmosphère séchante, régulée en température et en humidité relative. Étant donné la symétrie axiale et circonférentielle du problème, les équations du modèle $(1,2,3)$ sont résolues dans le domaine d'espace $\Omega=[0, R(t)] \times[0, h(t)]($ Fig. 6$)$.

\subsection{Formulation variationnelle de l'équation de transport de l'eau}

Le système d'équations $\Sigma_{1}$ à résoudre est le suivant :

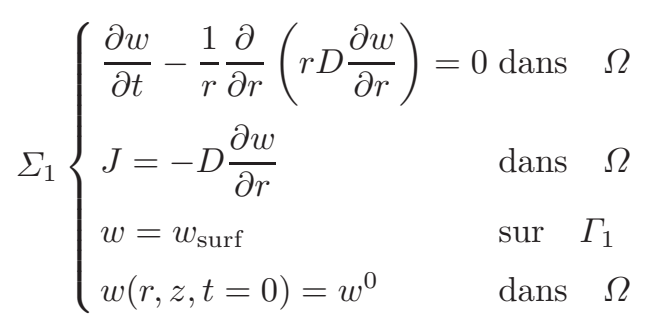

où la teneur en eau $w_{\text {surf }}$ est imposée par l'isotherme de désorption. 


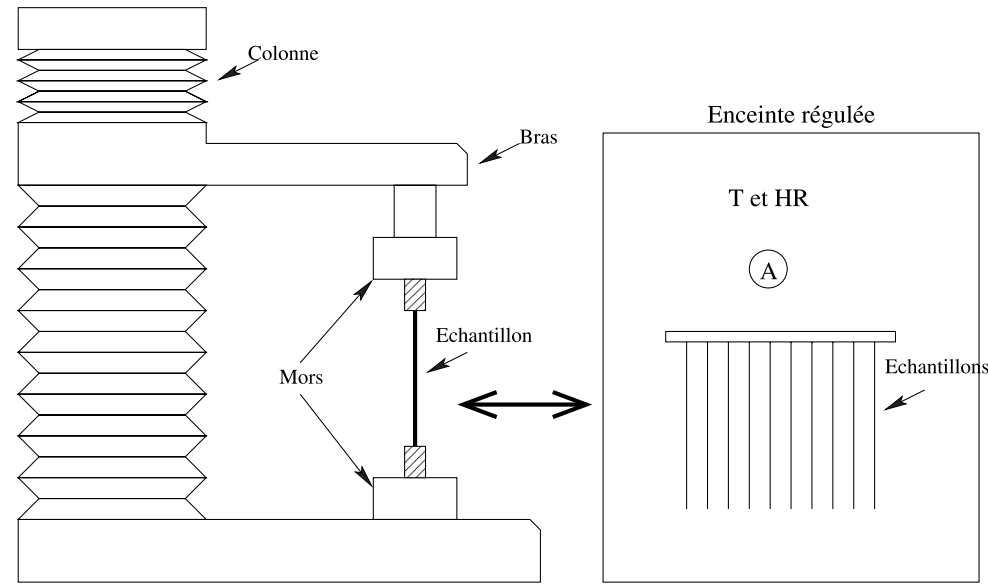

Fig. 3. Schéma de la machine d'essais mécaniques (RHEO TAXT 2/5).

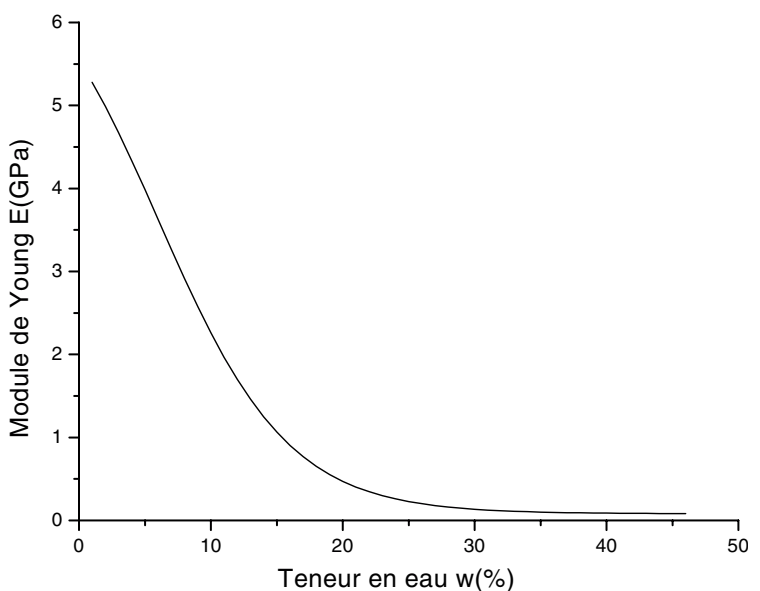

Fig. 4. Évolution du module de Young en fonction de la teneur en eau.

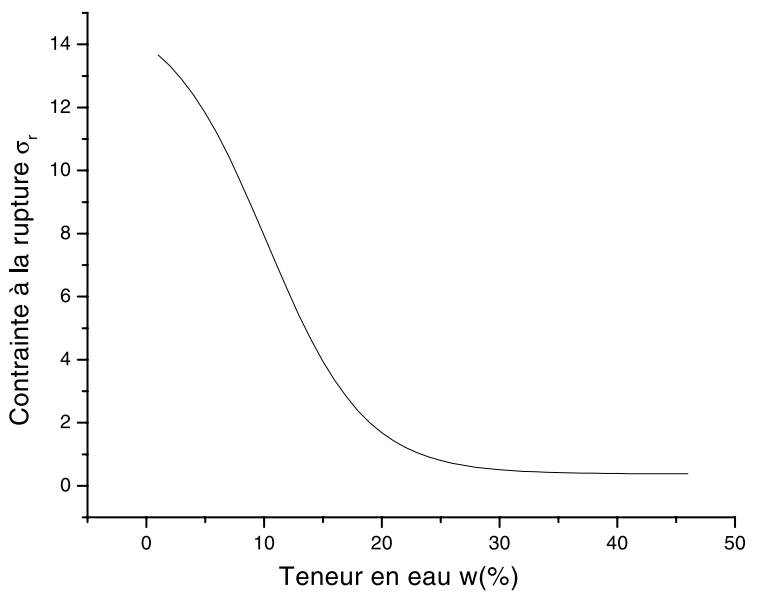

Fig. 5. Évolution de la contrainte à la rupture en fonction de la teneur en eau.

La formulation variationnelle de l'équation de transport de l'eau est de type Galerkin pour laquelle les valeurs virtuelles de $w$ sont prises comme des variations réelles

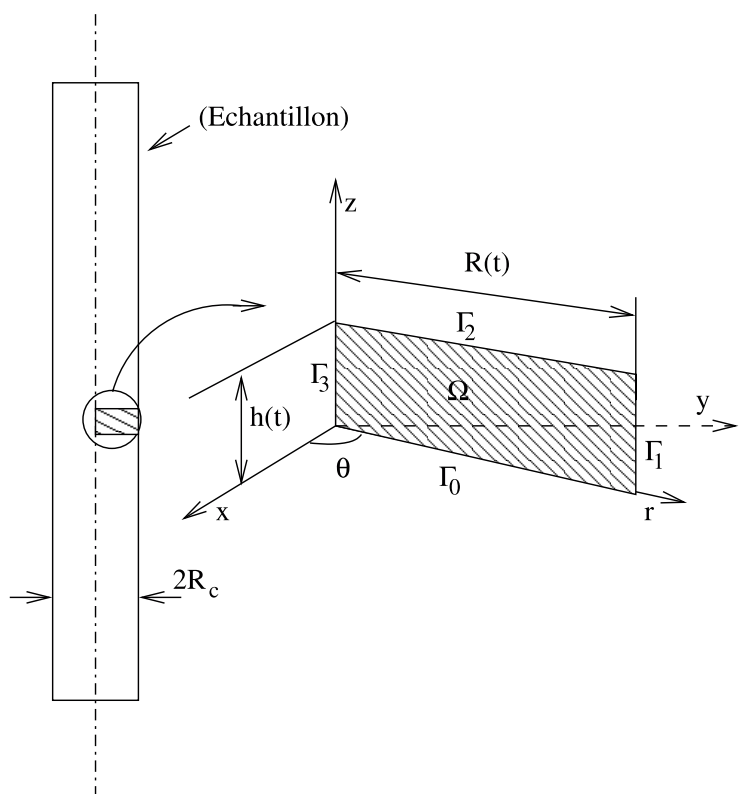

Fig. 6. Représentation schématique du milieu d'étude.

de $w$. En utilisant les conditions à la limites en teneur en eau et en flux, on obtient le système différentiel suivant [3] :

$$
[\mathbf{M}]\{\dot{\mathbf{w}}\}+[\mathbf{K}]\{\mathbf{w}\}=\{\mathbf{F}\}
$$

- $[\mathbf{M}]$ est la matrice de masse contenant les valeurs de $\rho_{\mathrm{s}}$;

- $[\mathbf{K}]$ est la matrice de rigidité contenant les valeurs du coefficient de diffusion;

- $\{\mathbf{F}\}$ est le vecteur forces extérieures contenant les valeurs de la teneur en eau à la surface;

- $\{\mathbf{w}\}$ est le vecteur contenant les valeurs nodales de la teneur en eau et $\{\dot{\mathbf{w}}\}$ sa dérivée partielle par rapport au temps. 


\subsection{Formulation variationnelle de l'équation d'équilibre mécanique}

Le problème mécanique est réduit au système d'équations aux dérivées partielles suivant :

$$
\Sigma_{2}\left\{\begin{array}{lc}
\operatorname{div}\left(\sigma_{i j}\right)=0 & \text { dans } \Omega \\
\sigma_{i j} \overrightarrow{\mathbf{n}}=0 & \text { sur } \Gamma_{1} \text { et } \Gamma_{2} \\
u_{r}(0, z, t)=0 & \text { sur } \Gamma_{3} \\
u_{z}(r, 0, t)=0 & \text { sur } \Gamma_{0} \\
u_{r}(r, z, t=0)=u_{z}(r, z, t=0)=0 \text { dans } \quad \Omega
\end{array}\right.
$$

Dans le cadre des déformations infinitésimales, le tenseur de déformation de Green-Lagrange $\epsilon_{i j}$ est défini par ses composantes :

$\epsilon_{r r}=\frac{\partial u_{r}}{\partial r} \quad \epsilon_{\theta \theta}=\frac{u_{r}}{r} \quad \epsilon_{z z}=\frac{\partial u_{z}}{\partial z} \epsilon_{r z}=\frac{1}{2}\left(\frac{\partial u_{r}}{\partial z}+\frac{\partial u_{z}}{\partial r}\right)$

Le tenseur des contraintes $\sigma_{i j}$ est lié au tenseur des déformation $\epsilon_{i j}$ par l'équation (2).

La formulation variationnelle du système $\Sigma_{2}$ est de type déplacements. Les grandeurs qui interviennent dans le problème d'équilibre sont des déplacements virtuels aux nœuds. La formulation variationnelle associée à l'équation d'équilibre (3) est de type Galerkin, pour laquelle les déplacements virtuels sont définis comme des variations des déplacements réels [3]. Comme pour l'équation de transport de l'eau, la discrétisation du système variationnel lié au système $\Sigma_{2}$ permet d'avoir le système matriciel suivant :

$$
[\mathbf{K}]\left\{\mathbf{u}_{\mathrm{n}}\right\}=\{\mathbf{F}\}
$$

- $[\mathbf{K}]$ est la matrice de rigidité contenant les valeurs du module d'élasticité aux nœuds ;

- $\{\mathbf{F}\}$ est le vecteur forces extérieures contenant les variations nodales de la teneur en eau $\delta w$;

- $\left\{\mathbf{u}_{\mathrm{n}}\right\}$ est le vecteur déplacements nodaux.

Les deux systèmes d'équations $\Sigma_{1}$ et $\Sigma_{2}$ sont résolus en utilisant la bibliothèque modulaire d'éléments finis, MODULEF [7].

\section{Simulation numérique et discussion}

\subsection{Conditions aux limites - Diagrammes de séchage}

La simulation numérique porte sur trois diagrammes de séchage qu'on dénomme D1, D2 et D3, pour des échantillons de diamètre initial $R_{\mathrm{c}}=1,7 \mathrm{~mm}$. On analysera l'influence de l'humidité relative $(H R)$ sur l'évolution des contraintes de séchage. Pour les trois diagrammes, l'évolution de la température donnée sur la figure 7 est identique. La donnée à chaque instant du couple $(T, H R)$ permet de calculer la teneur en eau d'équilibre $w_{e}$ à la surface par l'équation (4). La figure 8 donne l'évolution de la teneur en eau d'équilibre à la surface du matériau. Ces courbes sont utilisées comme conditions à la limite

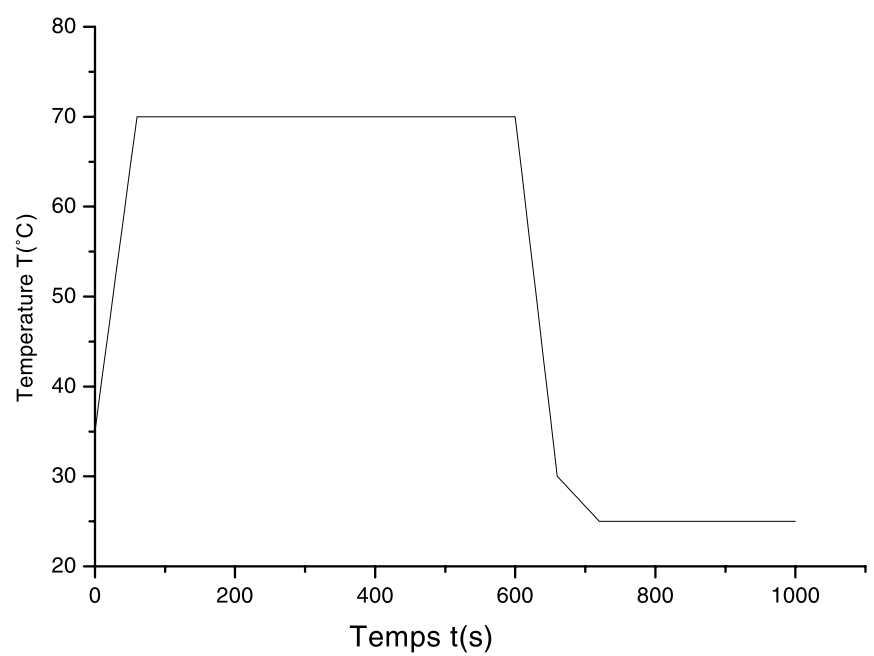

Fig. 7. Évolution de la température au cours d'un cycle de séchage

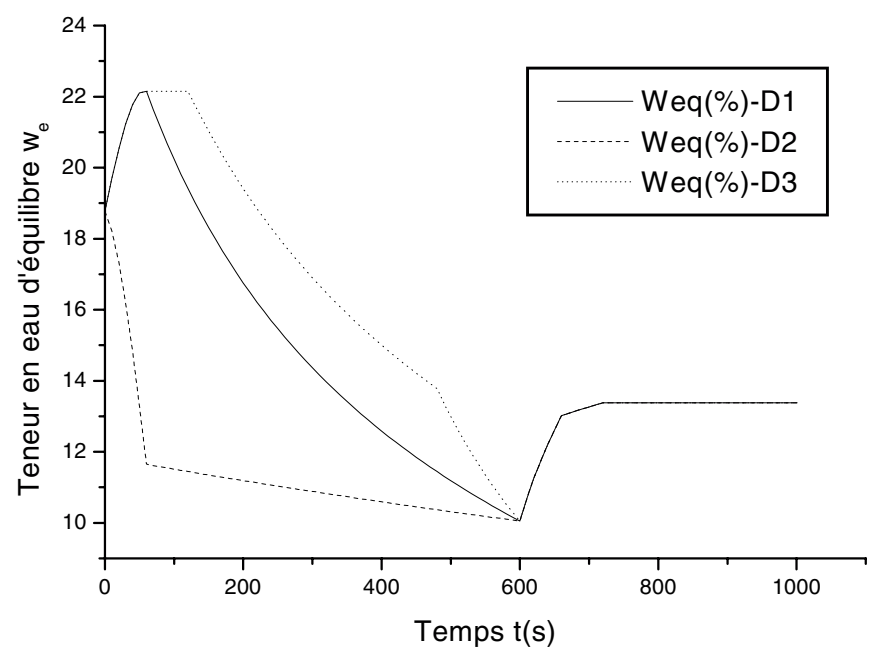

Fig. 8. Évolution de la teneur en eau à la surface du matériau.

pour le calcul des profils de teneur en eau dans le milieu. La phase de séchage correspond à l'intervalle de temps $[0,10 \mathrm{~h}]$, la phase de refroidissement correspond à l'intervalle $[10 \mathrm{~h}, 11 \mathrm{~h}]$ et la phase de stockage correspond à $t>11 \mathrm{~h}$.

\section{2 Évolution de la teneur en eau}

La symétrie axiale du problème de transport de l'eau impose que la teneur en eau varie uniquement en fonction de $r$ (Fig. 6). Les profils de teneur en eau sont représentés pour les diagrammes de séchage D1, D2 et D3 sur les figures 10-12. Sur ces figures, l'axe des abscisses représente le rayon $r(\mathrm{~mm})$ et l'axe des ordonnées représente la teneur en eau $w(\%)$. Ces figures montrent que pour les trois diagrammes de séchage, les profils de teneur en eau ne sont pas encore stabilisés à la fin du cycle de séchage. On remarque qu'une teneur en eau uniforme n'est atteinte que pendant la phase de stockage, environ $3 \mathrm{~h}$ après la fin du séchage. La valeur atteinte est d'environ $12,5 \%$. On 


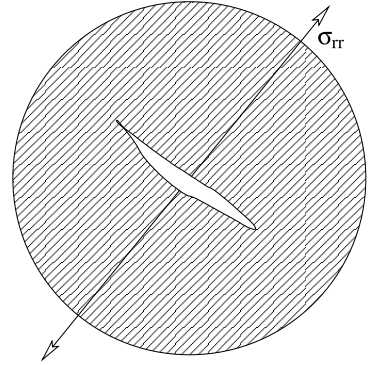

(a)

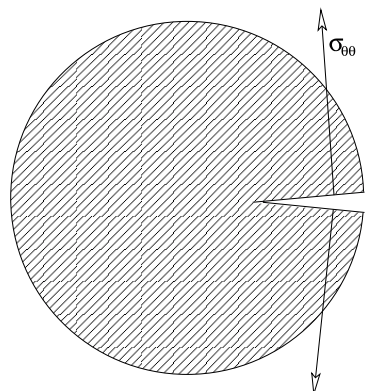

(b)

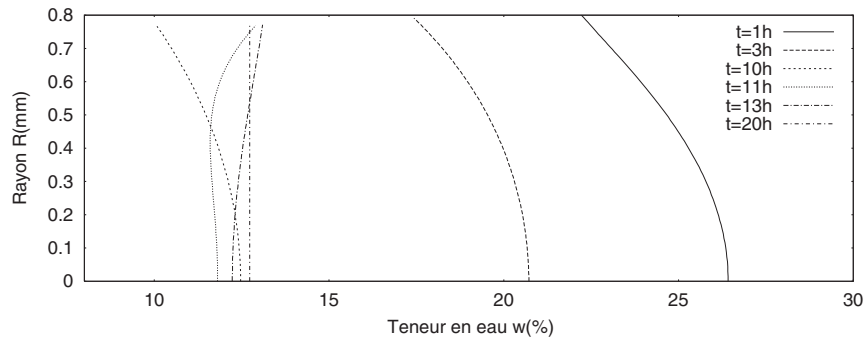

Fig. 10. Évolution des profils de teneur en eau du diagramme D1.

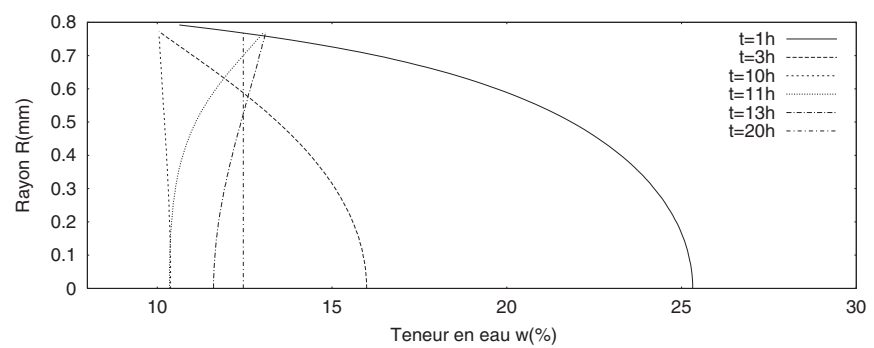

Fig. 11. Évolution des profils de teneur en eau du diagramme D2.

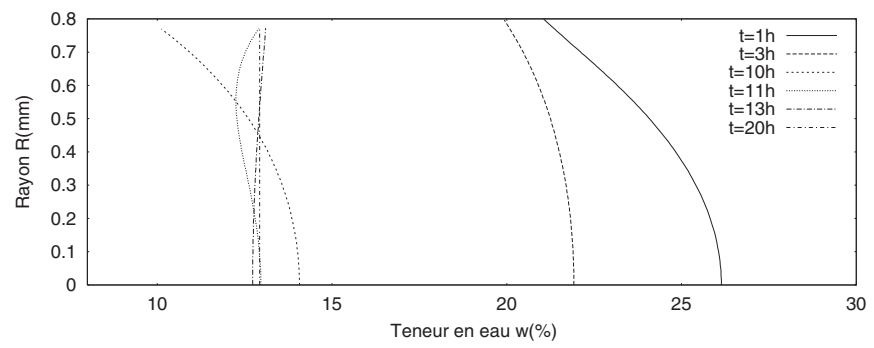

Fig. 12. Évolution des profils de teneur en eau du diagramme D3.

critique. En résumé, pour le diagramme D1, un risque de fissuration à cœur apparaît $3 \mathrm{~h}$ après le début du stockage mais l'état des contraintes tend à se stabiliser audessous de la valeur critique par la suite, limitant ainsi la propagation de la fissure. Au moment le plus critique, après $3 \mathrm{~h}$ de stockage, la zone de compression qui apparaît en périphérie pour la contrainte $\sigma_{\theta \theta}$ s'étend jusqu'à environ un rayon de $0,25 \mathrm{~mm}$. Cette zone tend à limiter le développement des fractures et les empêche de déboucher.

\subsubsection{Diagramme D2}

Au début du séchage (Fig. 16a) on voit apparaître une contrainte de traction $\sigma_{\theta \theta}$ à la surface du matériau. Cette contrainte reste très voisine de la contrainte de rupture. Il y a risque de fissuration en surface du type représenté sur la figure $9 \mathrm{~b}$. Cependant le dépassement constaté est très faible et on ne peut affirmer que ce type de fissure va apparaître. Une baisse accidentelle de l'humidité relative de l'air provoquerait certainement une fissuration de surface pendant le séchage pour ce diagramme. À la fin du séchage (Fig. 16c) une zone de traction apparaît dans la plus tard pendant la période de stockage (Figs. 13f et 14f les contraintes tendent à repasser au-dessous de la valeur
L'examen des figures $13 \mathrm{e}$ et $14 \mathrm{e}$ fait apparaître un dépassement de la contrainte de rupture au centre de l'échantillon sur les deux contraintes qui peut provoquer
une fissure de type présenté sur la figure 9a. Toutefois, séchage à un diamètre égal à 1,54 mm pendant la phase de stockage.

toutefois la dépasser. La contrainte $\sigma_{r r}$ reste en traction remarque une zone de traction dans la zone centrale et une zone de compression dans la partie périphérique du matériau. peut également constater sur ces figures la contraction du
matériau qui passe d'un diamètre de $1,7 \mathrm{~mm}$ en début de 

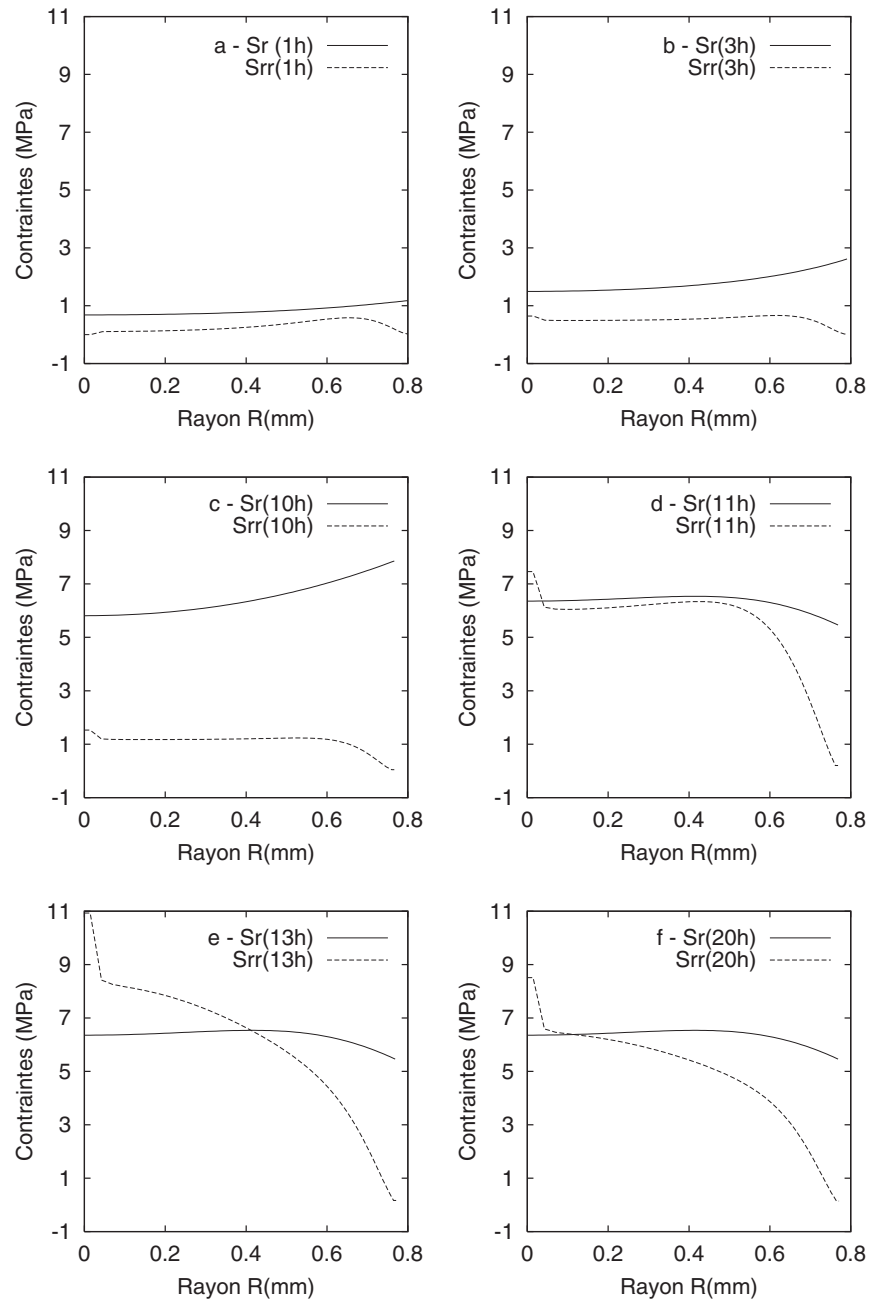

Fig. 13. Évolution de la contrainte radiale $\sigma_{r r}$ pour le diagramme D1.

zone centrale et la contrainte de rupture est dépassée. La contrainte atteint des valeurs très élevées, environ deux fois la limite de rupture. Pendant la période de stockage ce haut niveau de contrainte se maintient et une fissuration au cour du produit peut apparaître.

\subsubsection{Diagramme D3}

Les contraintes pendant la phase de séchage (Figs. 17a à $17 \mathrm{c}$ et $18 \mathrm{a}$ à $18 \mathrm{c}$ ) restent très faibles et aucun risque n'apparaît. À la fin de la phase de refroidissement (Figs. 17d et 18d) les contraintes restent inférieures à la contrainte de rupture. Pendant la phase de stockage (Figs. 17 e et $\mathrm{f}$ et $18 \mathrm{e}$ et $\mathrm{f}$ ) la contrainte radiale vient tangenter la contrainte de rupture au voisinage du centre sans toutefois la dépasser, il est de même de la contrainte tangentielle $\sigma_{\theta \theta}$. Ces figures montrent que non seulement les valeurs critiques ne sont pas atteintes mais que de plus (Figs. 18e et 18f) une zone de compression à la surface limiterait la propagation des fissures.
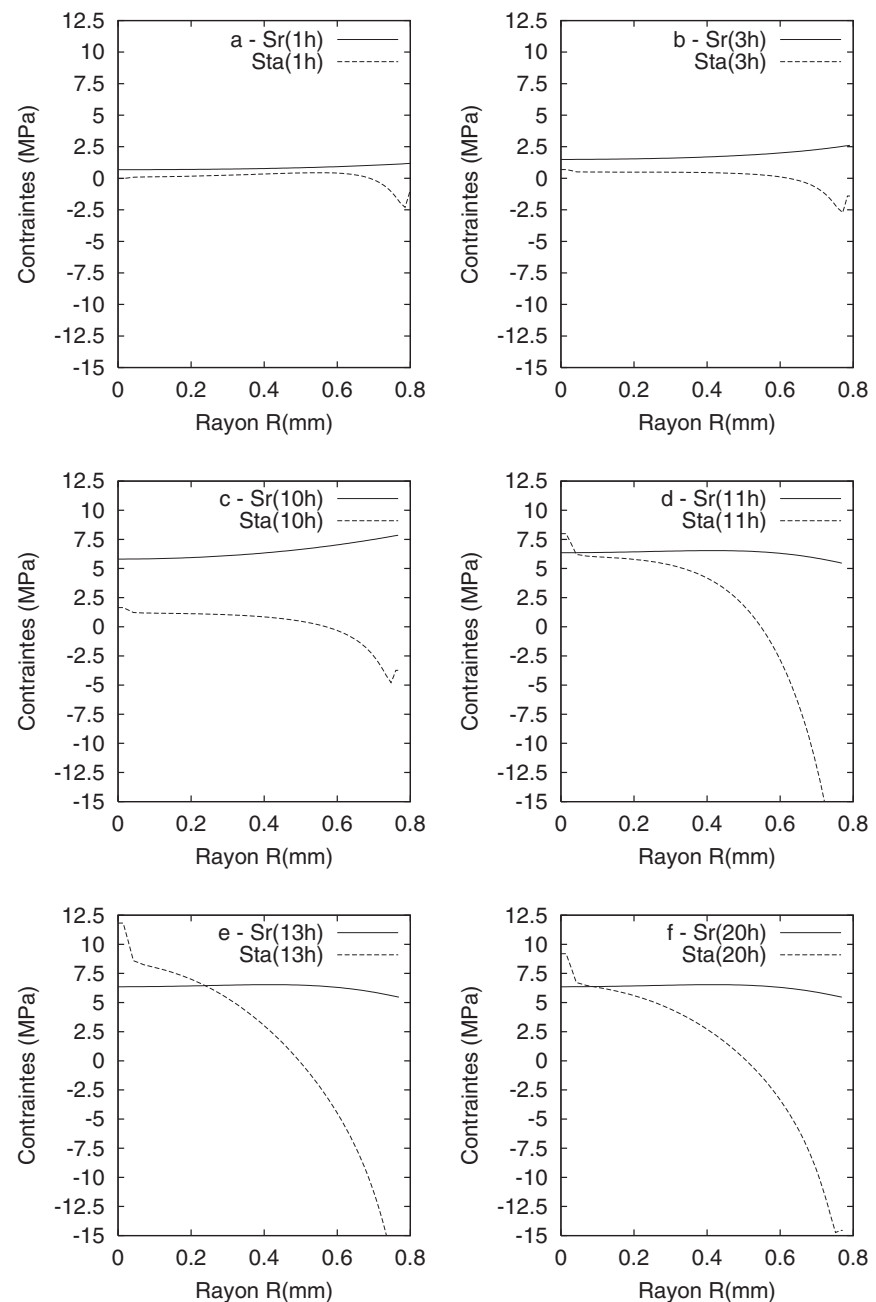

Fig. 14. Évolution de la contrainte tangentielle $\sigma_{\theta \theta}$ pour le diagramme D1.

Il apparaît à l'analyse des résultats que :

- Le diagramme D2 peut conduire à une fissuration de surface pendant le séchage et très certainement à la propagation d'une fissure centrale lors de la phase de stockage.

- Le diagramme D3 semble ne pas présenter de risque de fissuration.

- Le diagramme D1 présente une situation intermédiaire : la fissure semble exister cependant elle est contenue par la zone de compression périphérique et les risques de fissuration diminuent au cours du temps. Cependant toute manipulation du produit peut provoquer la rupture.

\section{Conclusion}

L'analyse des différentes simulations a permis de donner une explication cohérente du développement des fissures dans des matériaux pendant les processus de séchage, de refroidissement et de stockage. L'augmentation des niveaux de contraintes pendant la phase de 

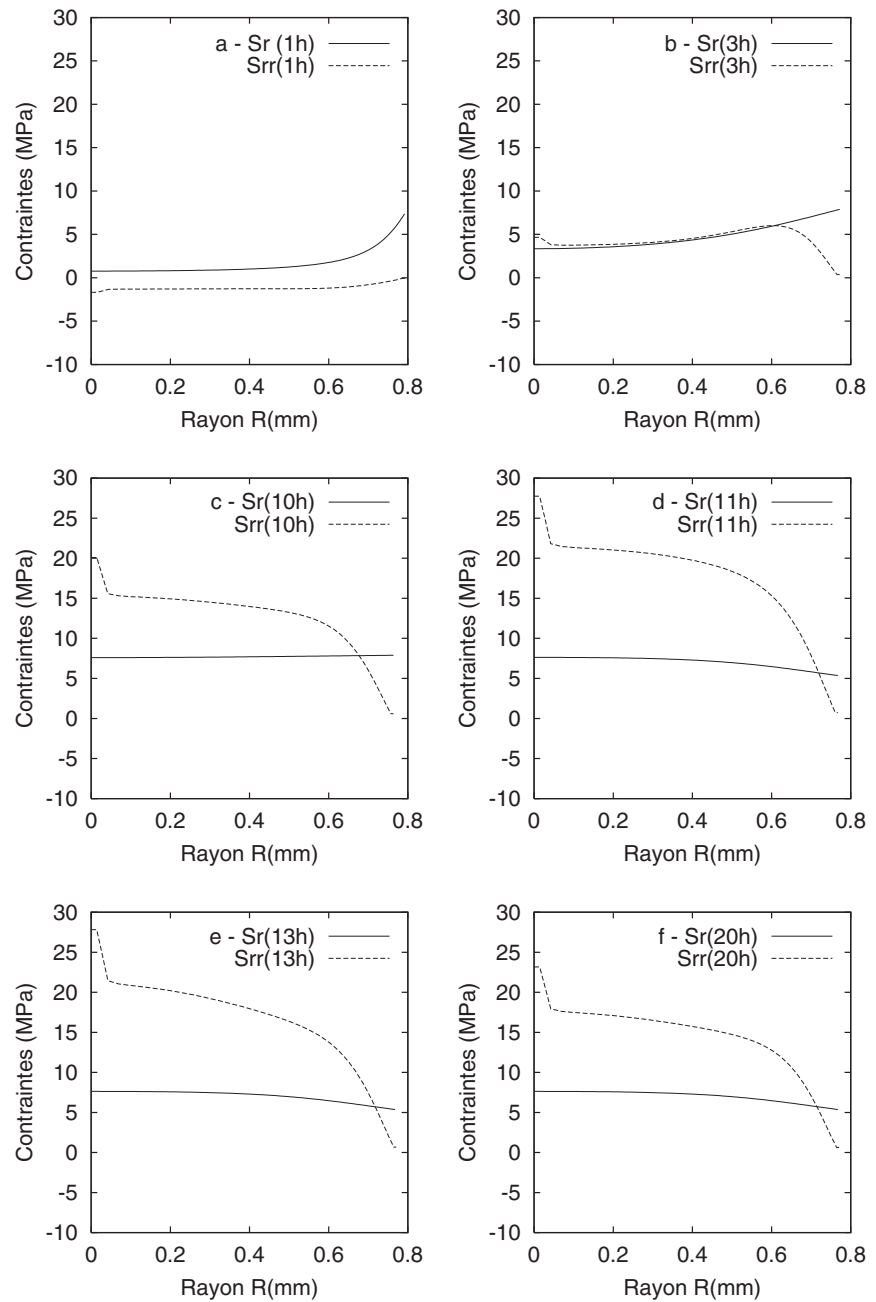

Fig. 15. Évolution de la contrainte radiale $\sigma_{r r}$ pour le diagramme D2.

stockage est la conséquence de la redistribution des profils de teneur en eau non stabilisés à la fin du séchage. Cette redistribution est accompagnée d'une rigidification de la partie centrale du matériau où les contraintes sont maximales. Ceci a pour conséquences l'accroissement du niveau des contraintes dans cette partie. Suivant les conditions imposées, ces fissures peuvent déboucher et provoquer une fissure pendant le stockage (diagramme D2) ou fragiliser le produit de telle sorte qu'une très légère sollicitation mécanique produise la rupture (diagramme D1). Les simulations numériques ont également montré qu'un trop faible niveau d'humidité relative peut provoquer une fissuration de surface pendant le séchage (diagramme D2).

Les diagrammes de séchage testés ont montré la forte sensibilité de l'état mécanique du produit dans ses différentes phases d'élaboration, à la régulation des paramètres de séchage : la température et l'humidité relative. Ceci est une conséquence directe des isothermes de désorption de la figure 1. À forte humidité relative (autour de $90 \%$ ) et quelle que soit la température, une très faible variation de l'humidité de l'air provoque une très forte variation de la teneur en eau à la surface ce qui
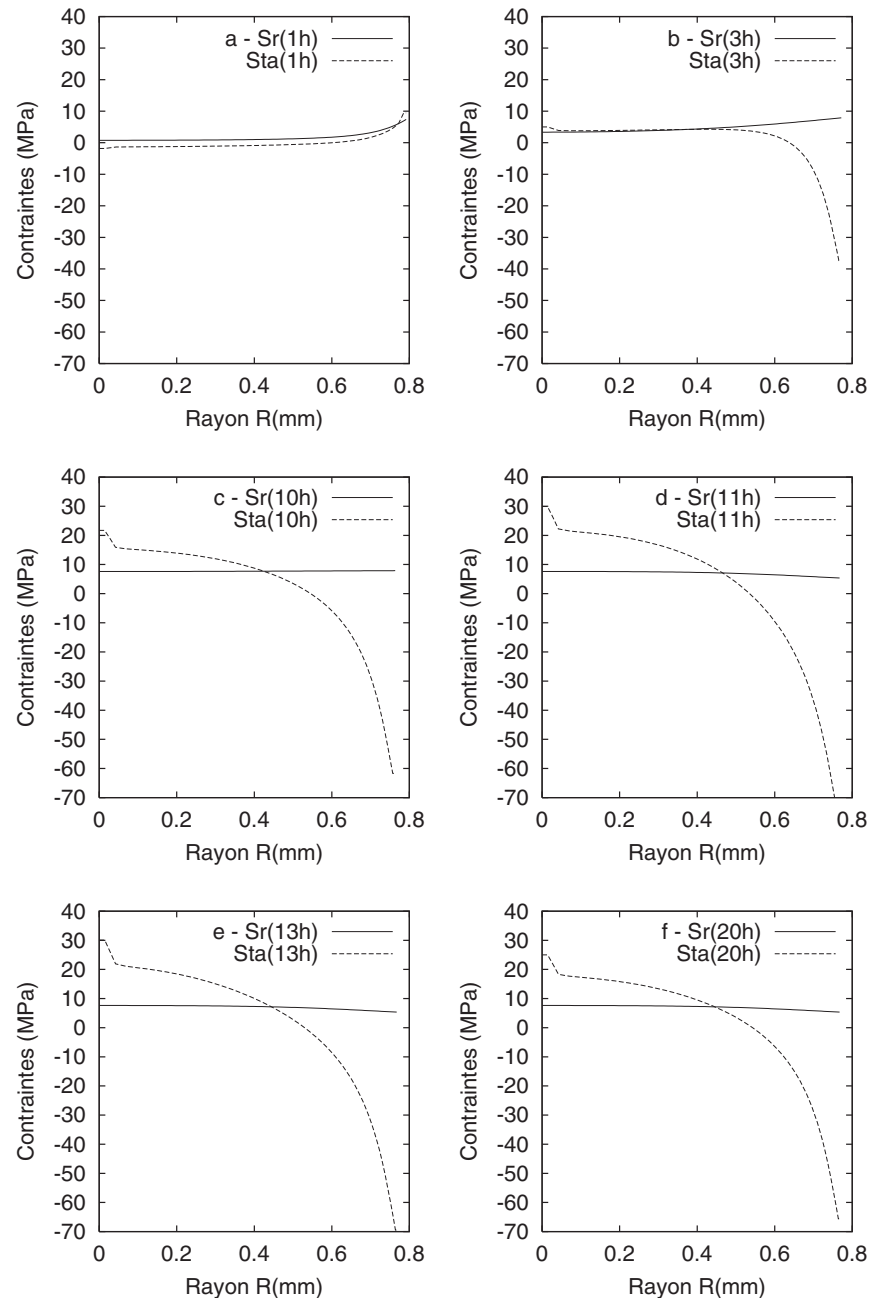

Fig. 16. Évolution de la contrainte tangentielle $\sigma_{\theta \theta}$ pour le diagramme D2.

induit des modifications importantes de la redistribution de l'eau dans le produit et donc de l'état des contraintes.

À l'humidité faible les isothermes sont très sensibles à la température; à titre d'exemple, à $40 \%$ le passage de $30{ }^{\circ} \mathrm{C}$ à $90{ }^{\circ} \mathrm{C}$ fait passer la teneur en eau de surface de $10 \%$ à $3 \%$. On voit sur la figure 4 que ceci correspond approximativement au doublement du module d'élasticité alors que la contrainte de rupture ne varie pas dans des proportions aussi larges.

La complexité des phénomènes mis en jeu ne peut être appréhendée qu'à travers le modèle numérique proposé. Celui-ci permet de rechercher les conditions optimales de séchage, de refroidissement et de stockage qui préservent la qualité du produit.

Remerciements. Ces travaux ont été réalisés avec l'appui technique de G. Denaeyer, assistant ingénieur au LMGC (UMR 5508). Ils ont été réalisés dans le cadre d'une convention entre le LMGC et la société Rivoire et Carret Lustucru. 

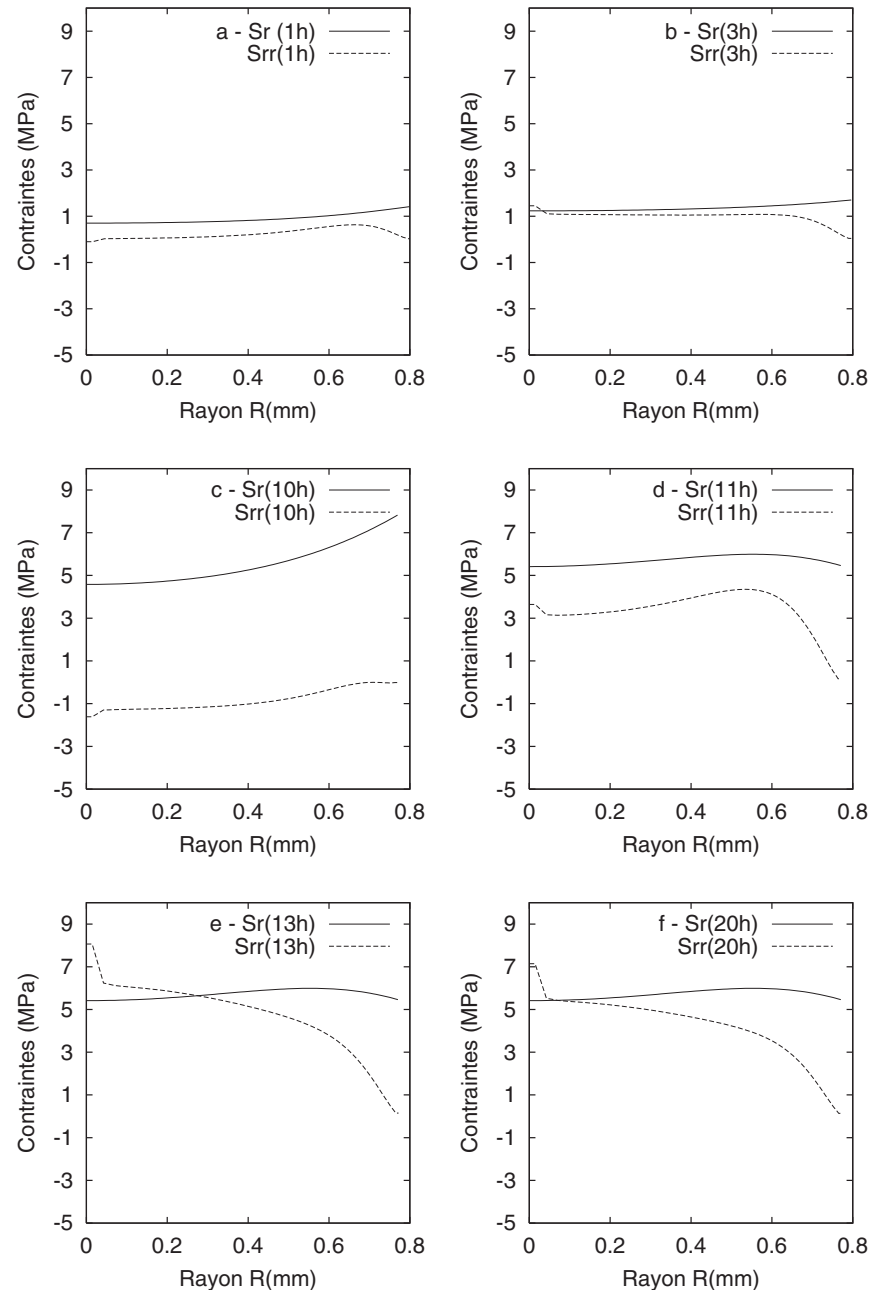

Fig. 17. Évolution de la contrainte radiale $\sigma_{r r}$ pour le diagramme D3.

\section{Références}

[1] I. Mrani, J.-C. Bénet, G. Fras, Transport of water in a biconstituant elastic medium, Appl. Mech. Rev., special issue on Mechanics of swelling 48 (1995) 717-721

[2] I. Mrani, G. Fras, J.-C. Bénet, Numerical study of dehydration stresses in agar gel, Drying Technology, special issue Mathema Techniques for the Solution of Dehydration Problems 13 (1995) 551-570

[3] I. Mrani, Comportement thermo-hygro-mécanique des produits fortement déformables : application à la maîtrise de la qualité lors du séchage, Thèse d'État, Université Cadi Ayyad, Marrakech, Maroc, 1997
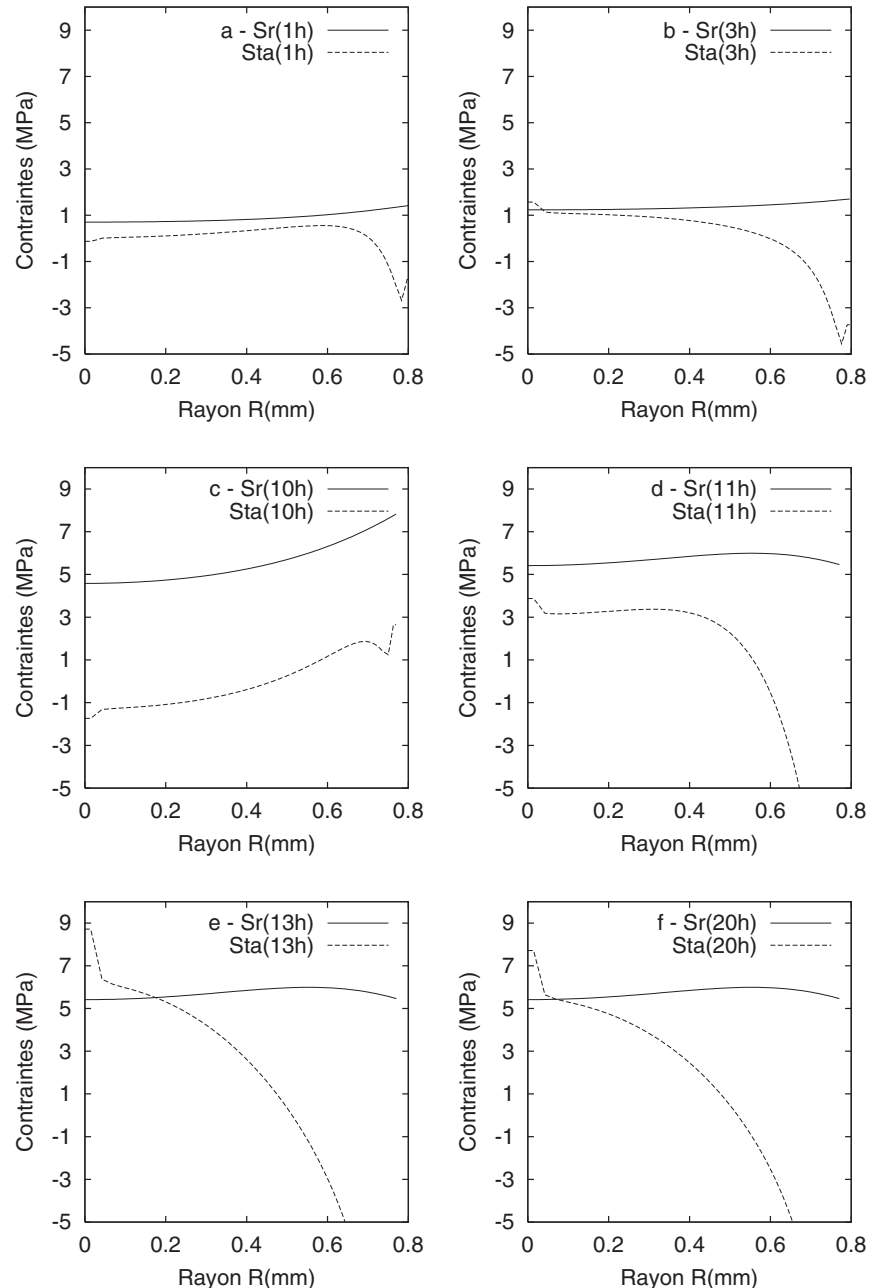

Fig. 18. Évolution de la contrainte tangentielle $\sigma_{\theta \theta}$ pour le diagramme D3.

[4] G. Ponsart, Caractérisation expérimentale d'un matériau alimentaire et modélisation des contraintes au cours du séchage : application aux pâtes spaghetti, Thèse, ENSIAA, France, 2002

[5] I. Mrani, Transport de matière en milieu biconstituant élastique : application au séchage du gel d'agar, Thèse, Université de Montpellier II, France, 1993

[6] J. Crank, The mathematics of diffusion, Oxford University Press, 1970

[7] M. Bernadou, P.L. George, Modulef une bibliothèque modulaire d'éléments finis, Éditions INRIA, France, 1988 